\title{
18-FDG PET/CT to reveal cardiac metastasis of pancreatic neuroendocrine cancer
}

\author{
Dariusz Jagielak ${ }^{1}$, Milosz Jaguszewski ${ }^{2}$, Jan Rogowski ${ }^{1}$, Renata Zaucha ${ }^{3}$ \\ ${ }^{1}$ Clinic of Cardiac and Vascular Surgery, Medical University of Gdansk, Poland \\ ${ }^{2} 1^{\text {st }}$ Department of Cardiology, Medical University of Gdansk, Poland \\ ${ }^{3}$ Clinic of Oncology and Radiotherapy, Medical University of Gdansk, Poland
}

A 49-year-old man was admitted to hospital complaining of intractable abdominal pain accompanied by bouts of diarrhea, flushing, and weight loss. Patient history indicated neuroendocrine pancreatic tumor (pNET) infiltrating Vater's papilla, duodenum and peripancreatic lymph nodes sealed by radical Whipple's operation. The inoperable multifocal relapse in the abdomen and pelvis

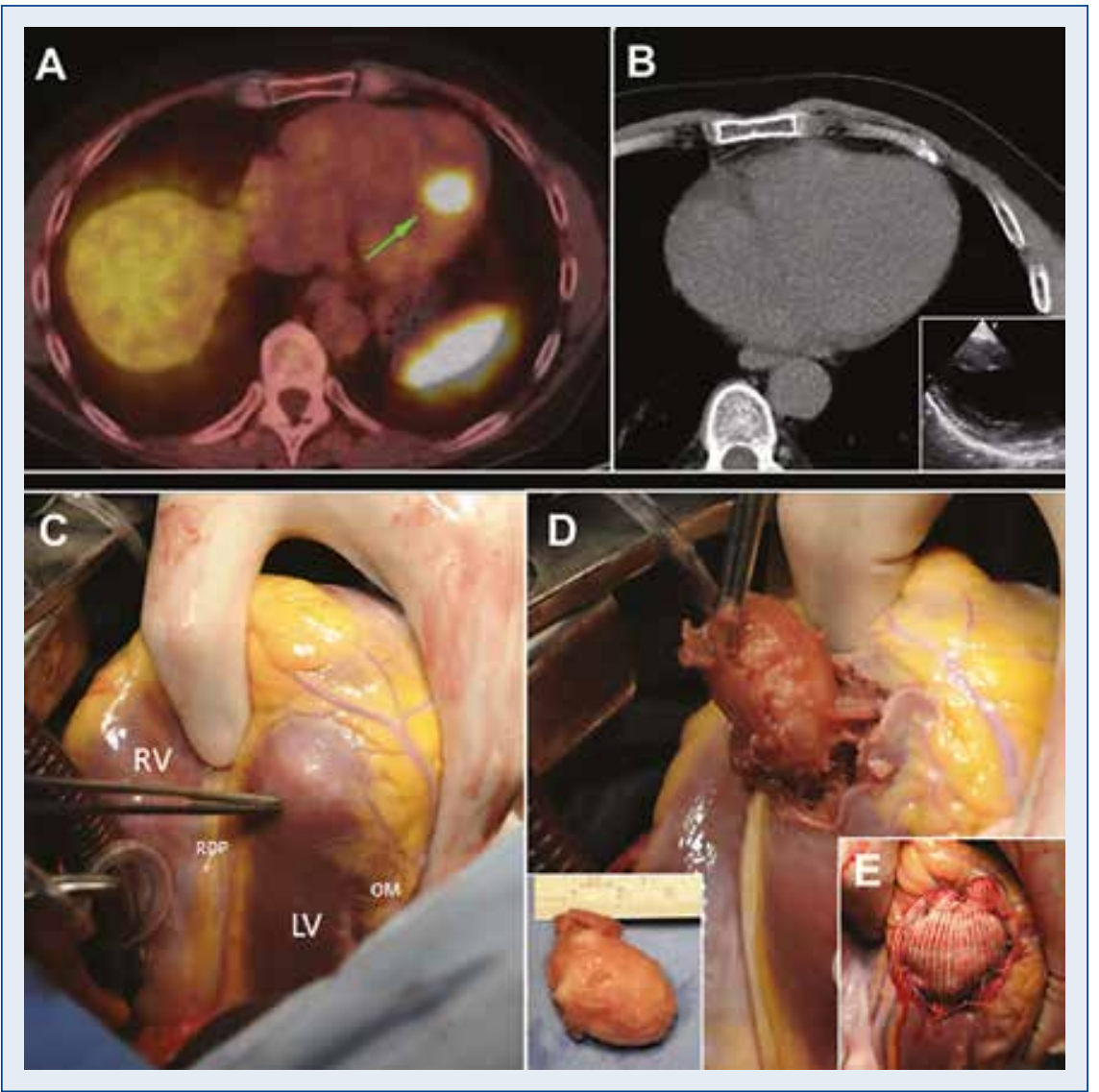

Figure 1. A. Radiotracer accumulated in the cardiac apex (green arrow). Cardiac apex in computed tomography and echocardiography with no pathological structures (B, inset). Surgical excision of the tumor (C, D); LV — left ventricle; $\mathrm{RV}$ - right ventricle; RDP — right posterior descendent; OM — obtuse marginal.

Address for correspondence: Dariusz Jagielak, MD, PhD, Department of Cardiosurgery, Medical University of Gdansk, ul. Dębinki 7, 80-291 Gdańsk, Poland, e-mail: darjag@gumed.edu.pl

Received: 11.10.2016 Accepted: 12.10 .2016 
was treated with 6 cycles of systemic chemotherapy, subsequent radical surgical excision of the residual masses and 9-month treatment with somatostatin analog. A scintigraphy performed 3 months prior to admission revealed a lesion located in close proximity to the superior mesenteric artery. The recurrent tumor was excised, but due to positive surgical margins, adjuvant radiotherapy was administered. Subsequent positron emission tomography (PET)/computed tomography (CT) performed at admission documented recurrent lesion close to mesenteric artery together with an intensive accumulation of the radiotracer in the cardiac apex (Fig. 1A) not apparent in CT (Fig. 1B) and echocardiography (Fig. 1B, inset). Due to highly improved performance status of the patient reporting only grade 1 diarrhea, cardiosurgical excision of a $5 \mathrm{~cm}$ tumor was successfully performed (Fig. 1C, D) and sealed by a sandwich Dacron patch (Fig. 1E). Due to expected progression of other in- operable abdominal metastatic lesions, the patient was offered everolimus given orally at daily doses of $10 \mathrm{mg}$, and proceeded with no evidence of intolerance. The postoperative course was uneventful, and the patient was discharged on the $6^{\text {th }}$ day post intervention.

Cardiac metastases from pNET belong to extremely unusual presentations of cancer progression. Cardiovascular symptoms are usually masked by other more prominent adverse events of advanced cancer. Up to $20 \%$ of cases may develop carcinoid heart disease with right-sided valve lesions or heart failure. So far, little is known about the clinical impact of cardiac metastasis on overall survival.

Herein was presented a unique case of a cardiac apex metastasis detectable only on 18-FDG $\mathrm{PET} / \mathrm{CT}$, which was treated successfully with radical surgical excision.

Conflict of interest: None declared 